\title{
Errata to 'Transitivity and invariant measures for the geometric model of the Lorenz equations'
}

\author{
CLARK ROBINSON \\ Department of Mathematics, Northwestern University, Evanston IL 60201, USA
}

(Received 1 November 1985)

\begin{abstract}
All the theorems stated in [2] are true. In particular, theorem B, on the existence of an invariant measure, is true. However, in the proof of theorem B, it is necessary to use the result of G. Keller [1] on the existence of an invariant measure for a $C^{1+\alpha}$ function. The attempt, in $\S 4$ of this paper, to modify the proof of S. Wong so that it applies to this general case of a $C^{1+\alpha}$ function, is faulty. These calculations do not overcome the real difficulties involved in the proof and leave major gaps in the estimates needed.
\end{abstract}

\section{REFERENCES}

[1] G. Keller. Generalized bounded variation and applications to piecewise monotonic transformations. Z. Wahrsch. Verw. Gebiete 69 (1985), 461-478.

[2] C. Robinson. Transitivity and invariant measures for the geometric model of the Lorenz equations. Ergod. Th. \& Dynam. Sys. 4 (1984), 605-611. 